\title{
Caracterização epidemiológica e clínica do HIV/Aids: associações com a mortalidade
}

\author{
Epidemiological and clinical characteristics of HIV/Aids: associations with mortality
}

Caracterización epidemiológica y clínica del VIH/Sida: asociaciones con la mortalidade

Leandra Fagan Rodrigues Gonçales ${ }^{1}$, Rafaela Marioto Montanha ${ }^{1 *}$, Renne Rodrigues ${ }^{1}$, Gilselena Kerbauy ${ }^{1}$, Rejane Kiyomi Furuya ${ }^{2}$, Natalia Marciano de Araujo Ferreira', Natacha Bolorino', Carla Fernanda Tiroli', Laís Cristina Gonçalves Ribeiro', Flávia Meneguetti Pieri'1.

\section{RESUMO}

Objetivo: Analisar o perfil epidemiológico e clínico dos casos de HIV/Aids e associar este perfil com a mortalidade. Métodos: Estudo transversal, realizado com dados das fichas de HIV/Aids do Sistema Nacional de Informação de Agravos de Notificação, de 2007 a 2019. Foi realizada análise de taxa de detecção de casos de HIV/Aids e calculada a razão de chances. Resultados: Dos 3.113 casos notificados, prevaleceram homens $(72,3 \%)$, faixa etária entre $30-59$ anos $(60,9 \%)$, com ensino médio completo $(39,5 \%)$ e raça/etnia branca $(67,3 \%)$. Houve aumento do percentual de homens $(p<0,001)$, diminuição da idade média $(p=0,012)$ e diminuição do percentual de óbito $(p<0,001)$. Indivíduos do sexo masculino, mais velhos, com menor escolaridade, heterossexuais e com infecções oportunistas apresentaram maior chance de óbito $(p<0,05)$. Conclusão: A associação entre baixa escolaridade e óbito demonstra a necessidade de educação em saúde de modo simples. A maior mortalidade em homens reforça a importância de políticas direcionadas a esse público.

Palavras-chave: Vírus da imunodeficiência humana, Epidemiologia, Mortalidade, Infecções oportunistas, Enfermagem.

\begin{abstract}
Objective: Analyze the epidemiological and clinical profile of HIV/Aids cases and associate this profile with the mortality. Methods: Cross-sectional study, using data from the HIV/Aids forms in the National Disease Notification System, between 2007 and 2019. The HIV/Aids case detection rate was analyzed and the odds ratio was calculated. Results: Among the 3,113 notified cases, men (72.3\%) prevailed, age range between 30 and 59 years $(60.9 \%)$, with finished secondary education $(39.5 \%)$ and white ethnic origin $(67.3 \%)$. The percentage of men increased $(p<0.001)$, while the average age $(p=0.012)$ and the percentage of deaths decreased $(p<0.001)$. Male and older individuals with lower education level, heterosexual and with opportunistic infections presented a greater chance of death $(p<0.05)$. Conclusion: The association between low education level and death demonstrates the need for simple health education. The higher mortality among men underlines the importance of policies targeting this public.
\end{abstract}

Keywords: Human immunodeficiency virus, Epidemiology, Mortality, Opportunistic infections, Nursing.

\section{RESUMEN}

Objetivo: Analizar el perfil epidemiológico y clínico de los casos de VIH/Sida y asociar este perfil con la mortalidad. Métodos: Estudio trasversal, desarrollado con datos de las hojas de VIH/Sida del Sistema Nacional de Información de Agravios de Notificación, de 2007 a 2019. Se analizó la tasa de detección de cases de VIH/Sida y se calculó el odds-ratio. Resultados: De los 3.113 casos notificados, prevalecieron hombres $(72,3 \%)$, rango de edad entre $30-59$ anos $(60,9 \%)$, con educación secundaria completa $(39,5 \%)$ y raza/origen étnico blanco $(67,3 \%)$. Aumentó el porcentaje de hombres $(p<0,001)$, mientras que disminuyeron

1 Universidade Estadual de Londrina (UEL), Londrina - PR.

*E-mail: rafaela.montanha@hotmail.com

2 Instituto Federal do Paraná (IFPR), Londrina - PR. 
el promedio de edad $(p=0,012)$ y el porcentaje de óbito $(p<0,001)$. Individuos del sexo masculino, más viejos, con menor escolaridad, heterosexuales y con infecciones oportunistas presentaron mayor chance de óbito $(p<0,05)$. Conclusión: La asociación entre escolaridad baja y óbito demuestra la necesidad de educación en salud de manera simple. La mayor mortalidad en hombres refuerza la importancia de políticas dirigidas a ese público.

Palabras-clave: Virus de la inmunodeficiencia humana, Epidemiología, Mortalidad, Infecciones oportunistas, Enfermería.

\section{INTRODUÇÃO}

A infecção pelo vírus da imunodeficiência humana e a síndrome da imunodeficiência adquirida (HIV/Aids) são considerados um problema de saúde pública global. Segundo dados da Joint United Nations Programme on HIV/Aids (UNAIDS) e da Organização Mundial da Saúde (OMS), mundialmente até 2018, as estimativas eram de 37,9 milhões de pessoas vivendo com HIV (PVHIV), sendo 1,7 milhões de casos novos anualmente. Desde o início da epidemia, 74,9 milhões de seres humanos foram infectados e 32 milhões tiveram o desfecho óbito (UNAIDS, 2019; WORLD HEALTH ORGANIZATION, 2016).

No Brasil, as estimativas de prevalência de infecção pelo HIV desde o início das notificações em 2007, por meio do Sistema de Informação de Agravo e Notificação (SINAN), foram de 300.496 casos de infecção pelo vírus (2007 a 2019). Em 2018, foram registrados pela Sistema de Informação de Mortalidade (SIM) um total de 10.980 óbitos por causa básica de Aids, com uma taxa de mortalidade de 4,4 por 100.000 habitantes. A taxa de mortalidade padronizada sofreu decréscimo de 22,8\% entre 2014 e 2018 (BRASIL, 2019).

Apesar da prevalência significativa do HIV/Aids, o perfil de mortalidade das PVHIV vem sendo alterado na era da terapia antirretroviral (TARV) (de PAULA AA, et al., 2020). Posto isto, é importante ressaltar que a mortalidade vem apresentando queda acentuada desde 1996, ano em que o Sistema Único de Saúe (SUS) passou a distribuir gratuitamente a TARV. Outros fatores também interferiram nesta mudança, como a descentralização do atendimento, que propicia um diagnóstico rápido e oportuno e o tratamento das infecções oportunistas (IOs), que interfere de forma positiva na sobrevida dos pacientes (LIMA RLFC, et al., 2017).

Em contraversão, a não adesão eficaz da TARV provoca uma imunodeficiência progressiva ao portador, com a supressão dos linfócitos T CD4+ e glóbulos específicos, reduzindo de forma gradual o potencial de defesa do sistema imune, tornado o paciente vulnerável as patologias oportunistas, mutações nos processos de divisão celular e infecções sistêmicas. Portando, a morbimortalidade do agravo está diretamente associada à imunodeficiência do paciente, em consequência da não adesão ou abandono da TARV ou por um diagnóstico tardio (SOUZA HC, et al., 2019). Dentre as infecções oportunistas mais recorrentes, encontra-se a tuberculose e pneumonia, complicações neurológicas em específico, a neurotoxoplasmose, candidíase oral, linfomas e sarcoma de Kaposi (SANTOS ACF, et al., 2020; CHAVES LL, et al., 2020; MUNIZ FCO, et al., 2018).

Com o objetivo de mudança no cenário descrito do HIV/Aids, afim de erradicar mundialmente a pandemia até 2030, a UNAIDS, projetou o plano da tríplice meta 90-90-90 com o propósito de: testar 90\% da população com HIV, tratar $90 \%$ dos casos positivos e manter $90 \%$ das pessoas em tratamento com carga viral (CV) não detectável. Com o alcance dessas metas, pelo menos $73 \%$ de todas as PVHIV no mundo estariam com supressão viral, em consequência haveria uma redução significativa de transmissão e aumento da sobrevida das PVHIV (BRASIL, 2017). No Brasil, segundo dados de 2018, 55\% das PVHIV tinham acesso a TARV e cerca de 60\% encontravam-se em supressão viral (UNAIDS, 2019).

A crescente complexidade e as mudanças de comportamento epidemiológico das PVHIV requerem o uso de indicadores diferenciados para conhecer os perfis epidemiológicos e clínicos dos acometidos por este agravo em cidades que apresentam altos índices da doença e do vírus.Para tanto, surgiu o seguinte questionamento: como se encontra o perfil epidemiológico dos casos de HIV/Aids no município de LondrinaPR e qual a sua associação com os casos das PVHIV que evoluíram para óbito? Dessa forma, o objetivo desse estudo foi analisar o perfil epidemiológico e clínico dos casos de HIV/Aids e associar estes perfis com a mortalidade. 


\section{MÉTODOS}

Trata-se de um estudo transversal retrospectivo, analítico exploratório, a partir de dados secundários das fichas de HIV/Aids do Sistema Nacional de Informação de Agravos de Notificação (SINAN). A população do estudo foi constituída pelos casos notificados na divisão de Vigilância Epidemiológica da Secretaria Municipal de Saúde que atendiam aos critérios de inclusão: casos notificados de PVHIV e Aids no período de 01 de janeiro de 2007 a 31 de dezembro de 2019, com idade igual ou superior a 13 anos, residentes no município de Londrina. Os dados foram coletados no período de novembro de 2018 a janeiro de 2020.

O município de Londrina está localizado no norte do estado do Paraná e é considerada a terceira maior cidade do sul do país. Em 2019, a estimativa populacional foi de 569.733 pessoas (IBGE, 2019). As variáveis incluídas na análise foram: sexo, idade, escolaridade, raça/cor, região do domicílio (zona urbana/periurbana ou rural), exposição (opção sexual), uso de drogas injetáveis, unidade de notificação, sintomas e IOs (definidos pelo Critério Rio de Janeiro/Caracas e Critério CDC), ano do diagnóstico e desfecho (óbito ou não óbito).

O cálculo da taxa média de detecção foi realizado para as variáveis sexo, idade, escolaridade, raça/cor e região do domicílio. A taxa de detecção por ano foi verificada empregando como denominador a população total do município obtida por meio de estimativas populacionais oriundas do Instituto Brasileiro de Geografia e Estatística (IBGE) para cada ano, realizando a médias dos anos ao final. As taxas foram calculadas por 100.000 habitantes (hab).

Para entender o comportamento de algumas variáveis contínuas ao longo do período de estudo, foi realizado o teste de Shapiro-Wilk para verificação da normalidade da taxa de detecção, percentual de casos do sexo masculino em cada ano, idade e taxa de mortalidade.

Variáveis normais foram testadas de acordo com a correlação de Pearson para identificação do coeficiente de correlação ( $r$ ) entres elas e o ano, e variáveis com distribuição não normal foram avaliadas por meio da correlação de Spearman (rho).

Para a análise de razão de chance considerou-se como variável dependente o desfecho (óbito e não óbito) e como variáveis independentes características sociodemográficas (sexo, idade, escolaridade, raça/cor, opção sexual e uso de drogas injetáveis) e relacionadas à presença de sintomas e IOs (candidíase oral, disfunção no sistema nervoso central - SNC, criptococcose extrapulmonar, pneumonia por Pneumocisti Jirovesi, toxoplasmose e linfócitos TCD4 $\leq 350$, caquexia, astenia e tosse persistente), utilizando regressão binária logística.

As variáveis que na análise bivariada apresentaram $p<0,20$ foram empregadas como ajuste no modelo. 0 nível de significância adotado foi de $5 \%$. A intensidade da associação foi determinada por meio da razão de chances - Odss Ratio (OR) e intervalos de confiança à 95\% (IC 95\%). As análises foram realizadas no programa IBM Statistical Package for the Social Sciences (SPSS) versão 20.0.0 estudo foi submetido e aprovado pelo Comitê de Ética em Pesquisa envolvendo Seres Humanos com CAAE: 00603718.6.0000.5231, número do Parecer: 2.978.859, atendendo às determinações da Resolução 466/2012.

\section{RESULTADOS}

Entre 2007 e 2019 foram notificados 3.113 casos de PVHIV, sendo majoritariamente do sexo masculino (72,3\%), faixa etária dentre $30-59$ anos $(60,9 \%)$, nível de escolaridade ensino médio completo $(39,5 \%)$ e a raça/cor branca (67,3\%) (Tabela 1). A taxa média de detecção anual foi de 44,63 por 100.000 hab., apresentando uma correlação com o ano de diagnóstico $(r=0,584, p=0,036)$, passando de $36,2 \mathrm{em} 2007$, para 79,2 por 100.000 hab. em 2019. O ano também se correlacionou com o aumento da porcentagem de casos em pessoas do sexo masculino ( $r h o=0,868, p<0,001)$, diminuição da idade média $(r=-0,672, p=0,012)$ e diminuição da porcentagem de óbito $(r=-0,948, p<0,001)$ que passaram, respectivamente, de $65,2 \%, 38,9$ anos e 8,5\% no triênio 2007-2009, para 78,2, 36,9 anos e 1,3\% no triênio 2016-2019. 
Tabela 1 - Caracterização dos casos notificados/confirmados de HIV/Aids no município de Londrina.

\begin{tabular}{|c|c|c|c|c|}
\hline Variável & $(\mathrm{N}=3.113)$ & $\%$ & População $^{a}$ & Taxa (100.000/hab) \\
\hline \multicolumn{5}{|l|}{ Sexo } \\
\hline Masculino & 2.250 & 72,3 & 243.059 & 71,21 \\
\hline Feminino & 863 & 27,7 & 263.642 & 25,18 \\
\hline \multicolumn{5}{|l|}{ Idade } \\
\hline $13-29$ & 1.052 & 33,8 & 170.694 & 47,41 \\
\hline $30-59$ & 1.896 & 60,9 & 205.815 & 70,86 \\
\hline$\geq 60$ & 165 & 5,3 & 64.476 & 19,68 \\
\hline \multicolumn{5}{|l|}{ Escolaridade $^{b}$} \\
\hline Analfabeto & 53 & 1,7 & 122.176 & 3,27 \\
\hline Ensino fundamental completo & 1.220 & 39,2 & 49.149 & 174,19 \\
\hline Ensino médio completo & 1.231 & 39,5 & 82.584 & 81,96 \\
\hline Ensino superior completo & 538 & 17,3 & 59.968 & 61,18 \\
\hline Ignorado & 71 & 2,3 & - & - \\
\hline \multicolumn{5}{|l|}{ Raça/cor } \\
\hline Branco & 2.095 & 67,3 & 356.990 & 45,14 \\
\hline Amarelos & 39 & 1,3 & 18.312 & 16,38 \\
\hline Não branco ou amarela & 977 & 31,4 & 131.321 & 57,23 \\
\hline \multicolumn{5}{|l|}{ Zona } \\
\hline Urbana/periurbana & 3.023 & 98,0 & 493.520 & 47,12 \\
\hline Rural & 62 & 2,0 & 13.181 & 36,18 \\
\hline Ignorado & 1 & 0,0 & - & - \\
\hline \multicolumn{5}{|l|}{ Exposição (Opção sexual) } \\
\hline Heterossexual & 1.863 & 59,9 & - & - \\
\hline Homem que faz sexo com homem & 956 & 30,7 & - & - \\
\hline Mulher que faz sexo com mulher & 2 & 0,1 & - & - \\
\hline Bissexual & 266 & 8,5 & - & - \\
\hline Ignorado & 26 & 0,8 & - & - \\
\hline \multicolumn{5}{|l|}{ Uso de Drogas Injetáveis } \\
\hline $\operatorname{Sim}$ & 77 & 2,5 & - & - \\
\hline Não & 2.980 & 95,7 & - & - \\
\hline Ignorado & 56 & 1,8 & - & - \\
\hline \multicolumn{5}{|l|}{ Unidade Notificadora } \\
\hline Hospitalar & 390 & 12,5 & - & - \\
\hline CTA & 2.261 & 72,6 & - & - \\
\hline UBS & 10 & 0,3 & - & \\
\hline $\begin{array}{l}\text { Divisão municipal de } \\
\text { epidemiologia }\end{array}$ & 2 & 0,1 & - & - \\
\hline Ignorado & 450 & 14,5 & - & - \\
\hline
\end{tabular}

Legenda: aEstimada com base em dados do censo de 2010, disponíveis no portal do IBGE, para cada grupo em particular. 'Para o cálculo da taxa foi levado em consideração apenas os casos de pessoas com 25 anos ou mais, haja visto ser essa a faixa etária do indicador do IBGE. CAT = Centro de Testagem e Aconselhamento (CTA). UBS = Unidade Básica de Saúde. Fonte: GONÇALES LFR, et al., 2020.

Dentre as variáveis sociodemográficas, o sexo masculino, idade de 30-59 anos e $\geq 60$ anos, analfabetismo e ser heterossexual apresentaram maior chance de evoluir à óbito (Tabela 2). A cada ano de vida, a chance de óbito na população de estudo aumentou em 3,9\% (IC95\%: 3,0-4,8; $p<0,001$ ), em regressão logística 
empregando a idade como variável contínua, e controlada por sexo, escolaridade, opção sexual e uso de drogas. A presença de IOs ou de sintomas apresentaram maior chance de óbito nas PVHIV, destacando-se disfunções no SNC (Tabela 3).

Tabela 2 - Análise bivariada das variáveis demográficas de casos de HIV/Aids atendidos nos serviços de saúde no município de Londrina, Paraná, Brasil, em relação à mortalidade.

\begin{tabular}{ccccccc}
\hline & \multicolumn{3}{c}{ Óbito } & \multicolumn{2}{c}{ Não óbito } & \multirow{2}{*}{ OR (IC95\%) } \\
\cline { 2 - 6 } Variável & $\mathbf{n}$ & $\%$ & $\mathbf{n}$ & $\%$ & $\mathbf{p}$-valor & \\
\hline Sexo & & & & & & \\
\hline Masculino & 294 & 13,1 & 1949 & 86,9 & 0,012 & $1,41(1,08-1,84)$ \\
Feminino & 121 & 14,0 & 741 & 86,0 & - & 1,00 \\
\hline Idade & & & & & & \\
\hline $13-29$ & 52 & 5,0 & 996 & 95,0 & - & 1,00 \\
$30-59$ & 315 & 16,6 & 1578 & 83,4 & $<0,001$ & $2,76(1,96-3,90)$ \\
$\geq 60$ & 48 & 29,3 & 116 & 70,7 & $<0,001$ & $5,64(3,49-9,12)$ \\
\hline
\end{tabular}

\section{Escolaridade}

\begin{tabular}{|c|c|c|c|c|c|c|}
\hline $\begin{array}{l}\text { Analfabeto ou ensino } \\
\text { fundamental completo }\end{array}$ & 249 & 19,6 & 1022 & 80,4 & $<0,001$ & $2,31(1,53-3,48)$ \\
\hline Ensino médio completo & 102 & 8,3 & 1127 & 91,7 & 0,339 & $1,23(0,80-1,88)$ \\
\hline Ensino superior completo & 39 & 7,3 & 497 & 92,7 & - & 1,00 \\
\hline \multicolumn{7}{|l|}{ Raça/cor } \\
\hline Branco ou amarelo & 293 & 13,8 & 1835 & 86,2 & - & 1,00 \\
\hline Não branco ou amarelo & 122 & 12,5 & 853 & 87,5 & 0,129 & $1,22(0,94-1,57)$ \\
\hline \multicolumn{7}{|l|}{ Opção sexual } \\
\hline Outras opções sexuais & 81 & 6,6 & 1138 & 93,4 & - & 1,00 \\
\hline Heterossexual & 316 & 17,0 & 1544 & 83,0 & $<0,001$ & $1,68(1,22-2,31)$ \\
\hline \multicolumn{7}{|l|}{ Uso de Drogas Injetáveis } \\
\hline Sim & 19 & 24,7 & 58 & 75,3 & 0,188 & $1,49(0,82-2,75)$ \\
\hline Não & 354 & 11,9 & 2618 & 88,1 & - & 1,00 \\
\hline
\end{tabular}

Legenda: *Regressão binária logística ajustada por sexo, idade (categórica), escolaridade, opção sexual e uso de drogas. Fonte: GONÇALES LFR, et al., 2020. 
Tabela 3 - Análise bivariada dos sintomas e das infecções oportunistas dos casos notificados/confirmados de HIV/Aids nos serviços de saúde no município de Londrina, Paraná, Brasil em relação à mortalidade.

\begin{tabular}{|c|c|c|c|c|c|c|}
\hline \multirow{2}{*}{ Variável } & \multicolumn{2}{|c|}{ Óbito } & \multicolumn{2}{|c|}{ Não óbito } & \multirow{2}{*}{ p-valor } & \multirow{2}{*}{ OR (IC95\%) } \\
\hline & $\mathrm{n}$ & $\%$ & $\mathbf{n}$ & $\%$ & & \\
\hline \multicolumn{7}{|c|}{ Sintomatologia de Tosse persistente } \\
\hline Sim & 135 & 38,2 & 218 & 61,8 & \multirow{2}{*}{$<0,001$} & $4,04(3,05-5,34-$ \\
\hline Não & 278 & 10,1 & 2469 & 89,9 & & 1,00 \\
\hline \multicolumn{7}{|c|}{ Caquexia } \\
\hline $\operatorname{Sim}$ & 139 & 26,4 & 387 & 73,6 & \multirow{2}{*}{$<0,001$} & $2,27(1,75-2,94)$ \\
\hline Não & 273 & 10,6 & 2300 & 89,4 & & 1,00 \\
\hline \multicolumn{7}{|l|}{ Astenia } \\
\hline Sim & 122 & 25,6 & 355 & 74,4 & \multirow{2}{*}{$<0,001$} & $2,24(1,72-2,93)$ \\
\hline Não & 291 & 11,1 & 2331 & 88,9 & & 1,00 \\
\hline
\end{tabular}

\begin{tabular}{ccccccc}
\hline Candidíase oral & & & & & & \\
\hline Sim & 102 & 30,8 & 229 & 69,2 & $<0,001$ & $2,77(2,07-3,70)$ \\
Não & 310 & 11,2 & 2459 & 88,8 & & 1,00 \\
\hline Disfunção no SNC & & & & & & \\
\hline Sim & 98 & 55,7 & 78 & 44,3 & \multirow{2}{*}{ (3), } & $7,77(5,41-11,15)$ \\
Não & 314 & 10,7 & 2609 & 89,3 & & 1,00 \\
\hline
\end{tabular}

Pneumonia por Pneumocisti Jiroveci

\begin{tabular}{|c|c|c|c|c|c|c|}
\hline Sim & 48 & 47,5 & 53 & 52,5 & \multirow{2}{*}{$<0,001$} & $5,35(3,37-8,52)$ \\
\hline Não & 362 & 12,1 & 2633 & 87,9 & & 1,00 \\
\hline \multicolumn{7}{|c|}{ Toxoplasmose } \\
\hline $\operatorname{Sim}$ & 56 & 44,4 & 70 & 55,6 & \multirow{2}{*}{$<0,001$} & $4,69(3,11-7,09)$ \\
\hline Não & 354 & 11,9 & 2617 & 88,1 & & 1,00 \\
\hline \multicolumn{7}{|c|}{ Criptococcose extrapulmonar } \\
\hline Sim & 32 & 54,2 & 27 & 45,8 & \multirow{2}{*}{$<0,001$} & $5,04(2,82-8,99)$ \\
\hline Não & 378 & 12,4 & 2659 & 87,6 & & 1,00 \\
\hline \multicolumn{7}{|c|}{ Linfócitos TCD4 ${ }^{+}<350$} \\
\hline Sim & 321 & 17,6 & 1506 & 82,4 & \multirow{2}{*}{$<0,001$} & $2,37(1,77-3,17)$ \\
\hline Não & 84 & 6,7 & 1179 & 93,3 & & 1,00 \\
\hline
\end{tabular}

Legenda: *Regressão binária logística ajustada por sexo, idade (categórica), escolaridade, opção sexual e uso de drogas; SNC- Sistema Nervoso Central. Fonte: GONÇALES LFR, et al., 2020.

\section{DISCUSSÃO}

Neste estudo, foram identificados os principais fatores associados à infecção por HIV/Aids e sua associação com a mortalidade: sexo masculino, grau de escolaridade, faixa etária, infecções oportunistas e sinais clínicos. As limitações de estudo estão relacionadas ao fato de a pesquisa ter sido desenvolvida em 
um único município, com impossibilidade de avaliação dos atestados de óbitos dos pacientes notificados. Entretanto, em razão ao longo período de avaliação e a opção da realização de um estudo censitário, acreditase que os resultados são robustos para a identificação do perfil analisado. $O$ estudo contribuiu para o avanço do conhecimento científico, ao revelar informações sobre o perfil epidemiológico de indivíduos com HIV/Aids e as associações deste perfil com a mortalidade.

A prevalência do sexo masculino entre os casos novos de HIV foi igualmente observada em estudos nacionais realizados, em que foram possíveis observar o perfil epidemiológico dos usuários, determinando que existe um número aumentado de casos HIV entre os homens (SANTOS ACF, et al., 2020; PEREIRA GMF, et al., 2018; NASCIMENTO EB, et al.,2018; TRINDADE FF, et al., 2019; SZWARCWALD CL, et al., 2016). Equiparados aos dados nacionais, foram registrados $69 \%(n=207.207)$ dos casos no sexo masculino, no período entre 2007 e junho de 2019. A razão de sexos para o ano de 2018 foi de 26 homens para cada dez mulheres (BRASIL, 2019). Outros estudos internacionais corroboram as informações anteriores, além de demonstrarem que 68\% dos óbitos relacionados ao HIV foram em homens (CABRERA S, et al., 2019).

Em relação à categoria de exposição, este estudo revelou que os homens heterossexuais apresentam alta prevalência de HIV e apresentam maior chance de evoluir à óbito em decorrência do agravo. Por não serem considerados grupo de risco em relação ao contágio pelo HIV, as políticas de prevenção em saúde são direcionadas à outras populações consideradas vulneráveis (KNAUTH DR, et al., 2020). Outros estudos nacionais se assemelham aos resultados obtidos ao apresentar que os heterossexuais são predominantes entre as notificações de HIV/Aids (SZWARCWALD CL, et al., 2016; PEREIRA GFM, et al., 2018; SANTOS CG, et al., 2019; FIGUÊREDO JUNIOR EC, et al., 2020).

Mesmo diante do contexto anterior sobre um aumento significativo de casos heterossexuais no decorrer dos anos, os resultados encontrados neste estudo fazem nos atentar ao contágio em homens que fazem sexo com homens (HSH), o que pode agravar as taxas de infecção por praticarem o coito anal receptivo, devido ao fato de existir no canal anal grande quantidade de células dendríticas e linfócitos (VERONESI $R$ e FOCACCIA P, 2015). Esse dado reforça o preocupante aumento de casos novos de HIV entre jovens HSH com idade abaixo de 25 anos, além das altas taxas de prevalência de esquecimento do uso de preservativo após o consumo de álcool e drogas (SKAATHUN B, et al., 2020).

Estudos revelam que a grande maioria dos HSH não participam de atividades em serviços de saúde e/ou grupos organizados ou ONG, além do tema prevenção não ser assunto entre a conversas entre amigos (GOMES RR, et al., 2017). Diante deste contexto, percebe-se a necessidade de divulgar informações e concentrar esforços sobre medidas preventivas, especialmente, o uso de preservativos, em prol do aumento da sensibilidade para o autocuidado, consequentemente melhorando a procura aos serviços de saúde para informações e prevenção.

Diante disso, este estudo reflete a imprescindibilidade de políticas de saúde direcionadas à esta população, visto que essa prevalência de infectados pelo HIV/Aids no sexo masculino, bem como sua heterossexualização, pode estar relacionada à presença de um comportamento de risco como o não uso de preservativos para a prática sexual, além de o homem ter mais relações extraconjugais e a autoconvicção de invulnerabilidade masculina, elevando a probabilidade de contágio da doença (TRINDADE FF, et al., 2019; MENEZES AM, et al., 2018; NASCIMENTO EB, et al.,2018).

Em relação à idade, ressaltou-se a representatividade da faixa etária de 30 a 59 anos com 60,9\% dos casos. Entretanto, é válido ressaltar o aumento expressivo de casos novos entre jovens de 13 a 29 anos, correspondendo a $33,8 \%$ dos casos notificados. Sabe-se que esta faixa etária é marcada pelas descobertas, curiosidades, intensas mudanças, dúvidas, o que os torna extremamente vulneráveis aos problemas relacionados à saúde. Dessa forma, alguns fatores levam esta parcela da população a suscetibilidade ao vírus HIV e ao consequente aumento da mortalidade (COSTA FCA, et al., 2020).

Estudo realizado por Correia D, et al. (2018) corrobora com o anterior, em que demonstra que o elevado número de casos novos entre adolescentes/jovens pode estar relacionado com a iniciação precoce da atividade sexual, bem como desconhecer o seu parceiro e, ainda, a não utilização de métodos de prevenção, fatores que elevam a vulnerabilidade de adolescentes/jovens em contrair o HIV. 
Outro estudo apontou que a maior parte dos indivíduos pesquisados apresentavam situação de vulnerabilidade frente ao contágio pelo HIV devido ao início precoce da vida sexual, utilização de preservativos de forma esporádica, variabilidade de parceiros, consumo abusivo de álcool e drogas, a fragilidade do autocuidado e o conhecimento insuficiente sobre as doenças sexualmente transmissíveis (DST) / Aids (MOREIRA PA, et al., 2019).

Salienta-se que a identificação dessa faixa etária no perfil de casos de HIV/Aids pode contribuir para o planejamento das ações de prevenções, com enfoque na utilização de preservativos, fazendo-se necessários esforços com intervenções mais resolutivas, apoiados pelos órgãos competentes e intuições educativas, além do apoio da atenção primária em saúde a partir de materiais informativos, educativos e didáticos com a finalidade de minimizar as taxas de infecção por HIV e outras DST nessa parcela da população, bem como promover o autocuidado e diminuir o estigma que envolve as necessidades sexuais dos jovens.

Nesse cenário, evidências indicam que, o aumento das taxas de infecção por HIV está relacionado ao grau de escolaridade do indivíduo. Estudos demonstram que a baixa escolaridade resulta em aumento do risco de exposição ao HIV. Além disso, indivíduos analfabetos ou com até o ensino fundamental completo apresentaram maior chance de óbito, quando comparados com aqueles que concluíram o ensino superior.

Em um estudo semelhante, a faixa etária mais acometida pela infecção do HIV foram os indivíduos que declararam ter 4 a 8 anos de estudo. Esse contexto se dá devido à baixa instrução e estímulos, associadas ao menor acesso de conhecimento à saúde sobre a transmissibilidade do vírus. Além do mais, o nível de escolaridade pode influenciar em maior acesso a informações inerentes à infecção pelo HIV e, sobretudo, melhores condições para conviverem com a sua condição sorológica (TRINDADE FF, et al., 2019; GALVÃO MTG, et al., 2015).

No que diz respeito às modalidades de exposição, houve um predomínio de indivíduos que referiram não fazer uso de drogas injetáveis. Nessa direção, a atual circunstância vem ao encontro da literatura que revela tendências ao longo dos anos, de diminuição do uso de drogas injetáveis como modo de transmissão (VERONESI R E FOCACIA P, 2015).

Atualmente, a transmissão sexual do vírus devido o sexo desprotegido (sexo vaginal, oral e/ou oral) tem tido significativo destaque, dado pelo início da atividade sexual cada vez mais precoce, além da multiplicidade de parceiros e o uso ocasional de preservativos, principalmente quando associados ao alto consumo de álcool (ALVES APB e RAMOS BA, 2019).

Em relação à mortalidade, vale a pena mencionar que existe forte associação entre a presença de IOs e sinais clínicos e o risco de evoluir à óbito por HIV. As doenças oportunistas estão intimamente ligadas ao HIV. Estas ocorrem devido a acometimento da imunidade celular causado pela doença (CHAVES LL, et al., 2020).

A distribuição gratuita dos medicamentos retrovirais pelo Sistema Único de Saúde (SUS) teve seu início em 1996 e, desde 2013, o sistema público de saúde garante tratamento para todas as PVHIV, independentemente da CV.

A ampliação desse programa provavelmente representa um dos principais fatores para a diminuição da mortalidade no período de estudo, associado a adequada adesão à TARV, que apresenta papel protetor contra o desenvolvimento das IOs, já que nos últimos anos a evolução da terapia antirretroviral, considerada de alta potência, tem proporcionado aumento importante das taxas de sobrevida dos PVHIV (BRASIL, 2018).

Em vista disso, neste estudo, pacientes que desenvolveram candidíase oral, disfunções no SNC e doenças respiratórias têm mais chances de evoluir à óbito por HIV. Dessa forma, é importante analisar que os resultados da pesquisa se assemelham a outros estudos como os de LOW A, et al. (2016) que afirma que a candidíase oral é considerada um dos primeiros sinais clínicos da Aids e acomete grande porcentagem dos indivíduos infectados pelo HIV, principalmente aqueles que ainda não iniciaram tratamento com os TARV.

Outro estudo realizado com 66 PVHIV com candidíase oral, em um hospital de ensino do centro-oeste do Brasil, verificou-se que lesões orais estavam associadas com níveis mais elevados de imunossupressão. 
Ainda neste estudo, das 45 espécies de Candida isoladas, 66,7\% foram Candida albicans (SPALANZANI RN, et al., 2018).

À vista disso, é válido mencionar que algumas IOs, como as disfunções do sistema nervoso central (SNC), caracterizadas por encefalopatias e demências associadas à Aids, são comumente encontradas e tendem a equivaler a altas taxas de mortalidade relacionada a doença (CHAVES LL, et al., 2020).

Nesse cenário, um estudo realizado por Sartori GP, et al. (2019) mostrou que aproximadamente metade dos pacientes com HIV/Aids participantes da pesquisa apresentaram transtornos neurocognitivos com causa multifatorial. Outro dado importante levantado diz respeito à deficiência diagnóstica por parte do profissional médico em identificar as disfunções cognitivas, além de não se referir ao problema como uma possível consequência da baixa adesão a TARV.

Em estudo realizado por Chaves LL, et al. (2020), uma porcentagem expressiva dos pacientes em estudo apresentou neurotoxoplasmose e doenças respiratórias como a tuberculose pulmonar, associadas com a baixa adesão da terapia antirretroviral e consequente diminuição da imunidade e aumento do risco de infecção do paciente HIV por los.

Diante deste contexto, percebe-se a necessidade da orientação pelos profissionais da saúde sobre a doença, tratamento medicamentoso, bem como seus possíveis efeitos colaterais, além de formular estratégias como grupos de adesão ou consultas individuais de modo que se possa sanar todas as dúvidas dos pacientes, para que dessa forma possa ser alcançado uma estreita adesão ao tratamento prescrito, diminuindo significativamente as $\mathrm{IOs}$, tendo como resultado a redução das taxas de mortalidade pela doença, melhorando o prognóstico e a qualidade de vida para as PVHIV.

\section{CONCLUSÃO}

A partir do estudo, duas recomendações são importantes: i) unir esforços para o desenvolvimento de ações que possibilitem a prevenção do HIV/Aids principalmente, na população que se apresentou em destaque sendo, adolescents e jovens adultos no município de Londrina no Estado do Paraná, oportunizando o diagnóstico precoce, o tratamento oportuno, redução dos casos de óbitos relacionados a IOs e o acompanhamento dos usuários; ii) desenvolver novos estudos principalmente na APS que permitam identificar os fatores determinantes das PVHIV , do abandono e do tratamento, oferecendo subsídios para a tomada de decisão em saúde.

\section{REFERÊNCIAS}

1. ALVES APB, RAMOS BA. Vulnerabilidade a transmissão sexual do vírus da imunodeficiência humana (HIV): representações sociais de Universitários indígenas do Instituto Insikiran de formação superior indígena. Revista Eletrônica Acervo Saúde, 2019; 11(8):1-9.

2. BRASIL MS. Secretaria de Vigilância em Saúde. Departamento de Doenças de Condições Crônicas e Infecções Sexualmente Transmissíveis - DCCI. Boletim epidemiológico HIV/AIDS, 2019:1-70.

3. BRASIL MS. Secretaria de Vigilância em Saúde. Departamento de Vigilância, Prevenção e Controle das Infecções Sexualmente Transmissíveis, do HIV/Aids e das Hepatites Virais. Protocolo Clínico e Diretrizes Terapêuticas para Manejo da Infecção pelo HIV em Adultos, 2018:1-412.

4. BRASIL MS. Secretaria de Vigilância em Saúde. Manual técnico de elaboração da cascata de cuidado contínuo do HIV, 2017:1-48.

5. CABRERA S, et al. Mortalidad por sida en Uruguay: perfil de las personas fallecidas en 2014. Revista Medica Del Uruguay, 2019; 35(3):181-192.

6. CHAVES LL, et al. Prevalência de infecções oportunistas em pacientes HIV positivos atendidos no Centro de Testagem e Aconselhamento (CTA) em município do Pará, em 2015 e 2016. Revista Eletrônica Acervo Saúde, 2020; 51:1-9.

7. CORREIA D, et al. Notificação de casos de HIV/AIDS em adolescentes portadores de HIV/AIDS no Nordeste: série Histórica entre os anos de 2004 a 2014. Revista Diálogos Acadêmicos, 2018; 7(1):73-83.

8. COSTA FCA, et al. Perfil informacional de uma população jovem a respeito da AIDS e suas consequências. Revista Eletrônica Acervo Saúde, 2020; Sup.(47):1-8.

9. DE PAULA AA, et al. Mortality profiles among people living with hiv/aids: Comparison between Rio de Janeiro and other federative units between 1999 and 2015. Revista Brasileira de Epidemiologia, 2020; 23:1-12. 
10. FIGUEIRÊDO JÚNIOR EC, et al. Perfil epidemiológico dos casos de Aids notificados no Brasil entre os anos de 2009 a 2019. Research, Society and Development, 2020; 9(9):1-18.

11. GALVÃO MTG, et al. Qualidade de vida e adesão à medicação antirretroviral em pessoas com HIV. Acta paulista enfermagem, 2015; 28(1):48-53.

12. GOMES RR DE FM, et al. Fatores associados ao baixo conhecimento sobre HIV/AIDS entre homens que fazem sexo com homens no Brasil. Caderno de Saúde Publica, 2017; 33(10):1-15.

13. IBGE. Instituto Brasileiro de Geografia e Estatística. Censo demográfico 2010: Informações completas. Londrina: IBGE, 2019. Disponível em: https://cidades.ibge.gov.br/brasil/pr/londrina/panorama. Acesso em: 3 set. 2020.

14. KNAUTH DR, et al. HIV/AIDS diagnosis in heterosexual men: still a surprise after more than 30 years of the epidemic. Caderno de Saúde Publica, 2020; 36(6):1-11.

15. LIMA RLFC de, et al. Estimativas da incidência e mortalidade por vírus da imunodeficiência humana e sua relação com os indicadores sociais nos estados do Brasil. Revista Brasileira De Ciências Da Saúde, 2017; 21(2):139-144.

16. LOW A, et al. Incidence of opportunistic infections and the impact of antiretroviral therapy among HIV-infected adults in low-and middle-income countries: a systematic review and meta-analysis. Clin Infect Dis, 2016; 62(12):1595-603.

17. MENEZES AMF, et al. perfil epidemiológico das pessoas soropositivas 8 para HIV/Aids. Rev enferm UFPE on line, 2018; 12(5):1225-32.

18. MOREIRA PA, et al. Vulnerabilidade ao HIV/Aids em adolescentes de uma escola pública no interior de Sergipe. Rev Fun Care Online, 2019; 11(4): 868-872.

19. MUNIZ FCO, et al. Pacientes críticos com HIV/Aids: fatores associados às complicações. Dissertação (Monografia no Curso de Pós-graduação em Terapia Intensiva e Alta Complexidade). Escola Bahiana de Medicina e Saúde Pública, 2018.

20. NASCIMENT EB, et al. Perfil epidemiológico dos pacientes soropositivos para HIV no centro de testagem e aconselhamento em um hospital municipal de Maracanaú. Revista Expressão Católica Saúde, 2018; 3(2): 57-63.

21. PEREIRA GFM, et al. Epidemiologia do HIV e Aids no estado do Rio Grande do Sul, 1980-2015. Epidemiologia e Serviços de Saúde: Revista Do Sistema Único de Saúde Do Brasil, 2018; 27(4):1-11.

22. SANTOS ACF, et al. Perfil epidemiológico dos pacientes internados por HIV no Brasil. Revista Eletrônica Acervo Saúde, 2020; Sup.(48):1-9.

23. SANTOS GC DOS, et al. Perfil epidemiológico de pessoas vivendo com HIV/AIDS em um município no interior do estado do Espírito Santo, Brasil. Revista Brasileira de Pesquisa Em Saúde/Brazilian Journal of Health Research, 2019; 21(1):86-94.

24. SARTORI GP, et al. Trastornos neurocognitivos en pacientes VIH positivos. Datos preliminares de una coorte prospectiva uruguaia. Revista Médica del Uruguay, 2019; 35(3):171-180.

25. SKAATHUN B, et al. Recent HIV Infection among men who have sex with men and transgender women in Tijuana. Revista de Saúde Pública, 2020; 54(82):1-11.

26. SOUZA HC, et al. Análise da adesão ao tratamento com antirretrovirais em pacientes com HIV / AIDS. Revista Brasileira de Enfermagem, 2019; 72(5):1361-1369.

27. SPALANZANI RN, et al. Clinical and laboratorial features of oral candidiasis in HIV-positive patients. Revista da Sociedade Brasileira de Medicina Tropical, 2018; 51(3):352-356.

28. SZWARCWALD CL, et al. Estimation of HIV incidence in two Brazilian municipalities, 2013. Revista de Saúde Pública, 2016; 50(55):1-10.

29. TRINDADE FF, et al. Perfil epidemiológico e análise de tendência de HIV/AIDS. Journal Health NPEPS, $2019 ; 4$ (1):153-165.

30. UNAIDS. Global AIDS update. 2019. Disponível em: https://unaids.org.br/. Acesso em: 1 set. 2020.

31. VERONESI R e FOCACCIA P. Tratado de Infectologia. 5 ed., São Paulo: Atheneu, 2015; 2380p. 